\title{
Histopathological findings in oesophageal carcinoma with and without preoperative chemotherapy
}

\author{
S J Darnton, S M Allen, CW Edwards, H R Matthews
}

\begin{abstract}
Aims: To investigate the pathological effects of preoperative chemotherapy on oesophageal carcinoma.

Methods: Qualitative and quantitative changes in oesophageal carcinoma after preoperative chemotherapy were assessed by examination of biopsy specimens before treatment and resected specimens.
\end{abstract}

Results: Of 13 patients with adenocarcinoma treated with 5-fluorouracil, adriamycin, and mitomycin (FAM), nine showed minor histological changes compared with 14 control cases. All 12 patients with squamous carcinoma treated with preoperative mitomycin, ifosfamide, and cisplatin (MIC) showed noticeable histological changes when compared with the 13 control cases. Changes included complete ablation $(n=1)$ and partial regression (n $=5$ ) of the tumour. A quantitative estimate of the proportion of tumour to stroma showed no difference between control adenocarcinomas and those treated with chemotherapy. There was, however, a significant reduction $(p<0.01)$ in the proportion of tumour to stroma in the treated squamous group compared with the controls. There was no relation between the degree of response in squamous carcinomas and the degree of differentiation of the tumour. Patients in which squamous carcinomas responded well, as assessed quantitatively, showed a tendency to better survival at one year.

Conclusions: Histopathological changes attributable to chemotherapy can be observed in oesophageal carcinoma. The response of squamous carcinoma to MIC is histologically more evident than that of adenocarcinoma to FAM. A quantitative technique may be useful in assessing the effect of chemotherapy in oesophageal squamous carcinoma.

Laboratory

S J Darnton

$S$ M Allen

Department of

Histopathology

C W Edwards

Department of

Thoracic Surgery

H R Matthews

East Birmingham Hospital, Birmingham B9 5ST

Correspondence to: Dr S J Darnton Accepted for publication 15 July 1992 chemotherapy trials have used various drug regimens with assessment in terms of reduction in tumour size and survival, though the effect on long term survival is still uncertain
The pathological effects of preoperat chemotherapy in oesophageal carcinoma been the subject of review, ${ }^{1}$ but detail morphological descriptions of change follow-
At this hospital the results of studies on the effects of chemotherapy suggest that there is often a significant histological response which cannnot be detected by barium swallow or computed tomography. ${ }^{6}$ In this study we have attempted to quantitate the histopathological changes following preoperative chemotherapy.

\section{Methods}

Between January 1988 and July 1990, 55 consecutive patients with localised oesophageal carcinoma were treated by resection, with or without preoperative chemotherapy, and divided into four groups as follows.

Group 1 comprised 13 patients (12 male, mean age $62 \cdot 8$ years, range $45-71$ ) with adenocarcinoma of the lower oesophagus or oesophagogastric junction who received two preoperative pulses of 5-fluorouracil (David Bull Laboratories, Warwick, England), adriamycin (Doxorubicin, Farmatalia Carlo Erba Ltd, St Albans, England), and mitomycin (Mitomycin-C Kyowa, Martindale Pharmaceuticals Ltd, Romford, England) (FAM) with an interval of three weeks, followed by resection three weeks later. $^{7}$

Group 2 comprised 14 patients $(11$ male, mean age 63.6 years, range $45-76$ ) also with adenocarcinomas, but who received no preoperative chemotherapy because of dysphagia resistant to dilatation $(n=4)$, age above 75 years $(n=3)$, refusal $(n=3)$ and specific contraindications to chemotherapy $(n=4)$.

Group 3 comprised 12 patients (nine male, mean age 65.3 years, range 55-75) with squamous carcinoma of the oesophagus who received two preoperative pulses of mitomycin, ifosfamide (Mitoxana, Boehringer Ingelheim Ltd, Bracknell, England) and cisplatin (Lederle Laboratories, Gosport, England) (MIC) with an interval of three weeks, with resection three weeks later. ${ }^{8}$

Group 4 comprised 13 patients (three male, mean age $67 \cdot 1$ years, range $44-86$ years) also with squamous carcinoma but who received no preoperative chemotherapy because of dysphagia resistant to dilatation $(n=5)$, age above 75 years $(n=4)$, refusal $(n=1)$ and specific contraindications to chemotherapy $(n=3)$.

Pretreatment endoscopic biopsy and resected specimens were examined in all patients following fixation in $10 \%$ formol saline and staining with haematoxylin and eosin. Resected specimens were opened longitudinally, pinned out, and mapped prior to processing and three to five full thickness blocks of the 
tumour were taken and embedded in paraffin wax. Sections were cut at $3 \mu \mathrm{m}$ thickness. Pathological staging of tumour differentiation and invasion and node status was made according to the UICC classification. ${ }^{9}$

The following comparisons were then made:

(a) Pretreatment biopsy and resected specimens in patients treated with chemotherapy (groups 1 and 3).

(b) Preoperative biopsy and resected specimens in patients not treated with chemotherapy (groups 2 and 4 ).

(c) Resected specimens in group 1 (adenocarcinomas with chemotherapy) and group 2 (adenocarcinomas without chemotherapy).

(d) Resected specimens in group 3 (squamous carcinomas with chemotherapy) and group 4 (squamous carcinomas without chemotherapy).

In addition to a qualitative assessment, a quantitative morphometric measurement was made on the resected specimens to determine the proportion of tumour to stroma. A section was selected from the central area of each tumour, or in the case of grossly ablated tumours, from the centre of the healed area. The densest region of tumour compared with background stroma was located empirically and a submucosal field observed at a magnification of $\times 100$. A camera lucida permitted simultaneous observation of a movable stylus on a bit pad. The proportion of tumour area to stroma was measured using the digitising tablet system and stylus (Summagraphics Bit Pad Two, Summagraphics Corp., Fairfield, Connecticut, USA). Areas within the field were outlined by the stylus whose position and movement were recorded with the aid of a microprocessor (Acorn Archimedes 440/1 Computer, Acorn, Cambridge, England) and the appropriate software ("Digit Analysis Package", BP Hayes Software, Berkhamsted, England). The proportion of total tumour area to the area of background stroma (the area of the microscopic field) was then expressed as a percentage.

The mean and standard errors of these percentage values were taken for each patient group. Student's $t$ test on unpaired data was used to test values for groups 1 and 2, with populations of a normal distribution, at the 95\% confidence level. Wilcoxon's rank sum test for non-parametric unpaired data was used to test the values for groups 3 and 4, because the distribution in group 3 was not normal at the $95 \%$ confidence level.

\section{Results}

Qualitative changes seen after chemotherapy included complete ablation or partial regression of the tumour, regeneration of surface epithelium over previously ulcerated tumour areas, pronounced fibrosis and a chronic inflammatory reaction (figs 1 and 2).

(a) Pretreatment biopsy and resected specimens in nine adenocarcinomas treated with FAM 9 (out of 13 in group 1) showed only minor histological differences. In all 12 squa-

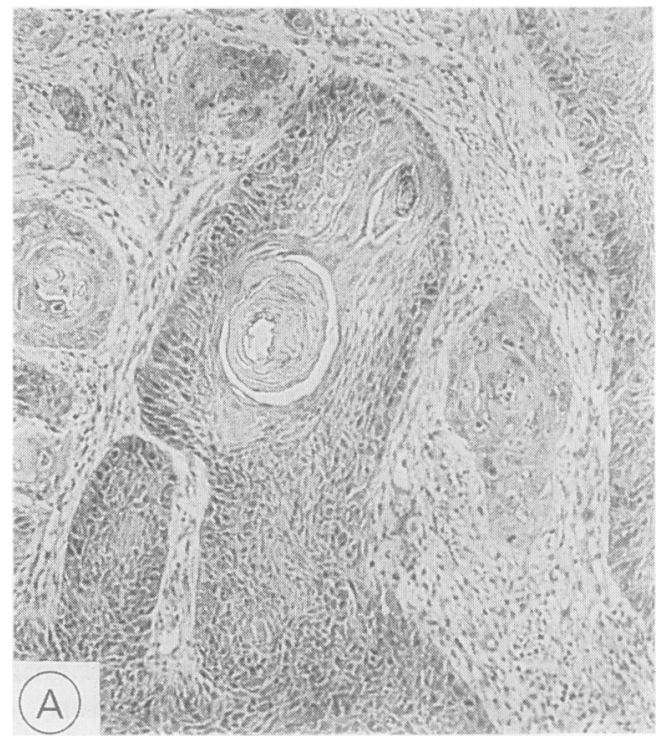

Figure 1A Case A: well differentiated squamous carcinoma. Pretreatment biopsy specimen.

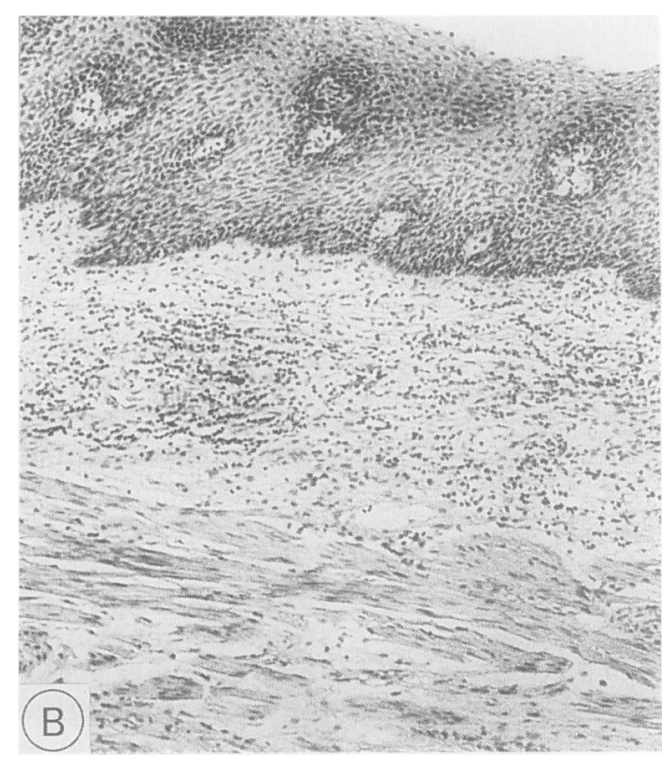

Figure $1 B$ Case $A$ : resected specimen following chemotherapy (MIC). There is complete ablation of tumour with re-epithelialisation, local chronic inflammation, and submucosal fibrosis.

mous carcinomas, however, there were pronounced differences between pretreatment biopsy and resected specimens consisting of ablation or regression of tumour, disappearance of surface ulceration with healing of the epithelium and fibrosis, and areas of chronic inflammatory exudate localised around tumour islands when present.

(b) Pretreatment biopsy and resected specimens in patients not treated with chemotherapy (groups 2 and 4) showed no histological differences.

(c) Comparison of resected specimens in groups 1 (adenocarcinomas treated with FAM) with resected specimens in group 2 (untreated adenocarcinomas) showed that nine out of 13 tumours in group 1 showed minor histological differences while the other four were identical in appearance.

(d) Comparison of resected specimens in group 3 (squamous carcinomas treated with 
Figure $2 A$ Case B: well differentiated squamous carcinoma. Pretreatment biopsy specimen.

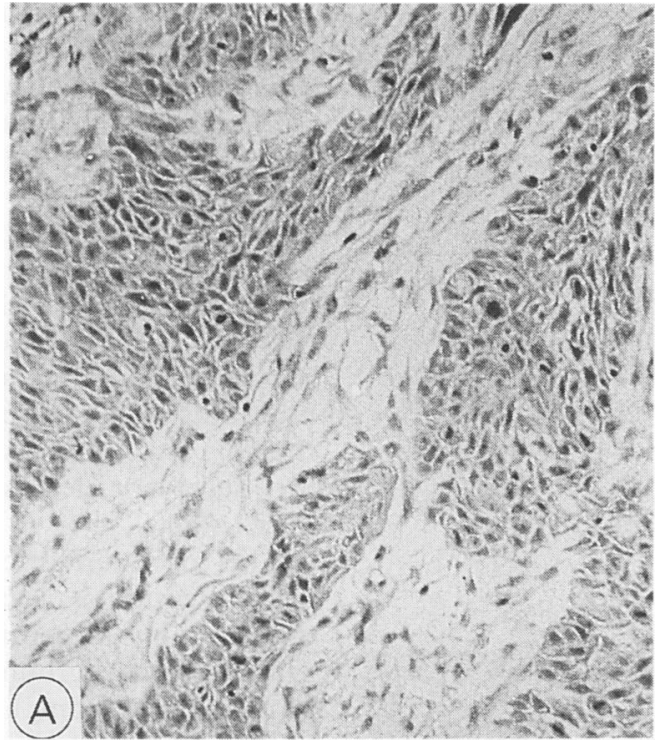

Figure 2B Case B: resected specimen following chemotherapy (MIC). There is pronounced regression of tumour to small foci of tumour cells (arrowed) surrounded by a chronic inflammatory exudate.

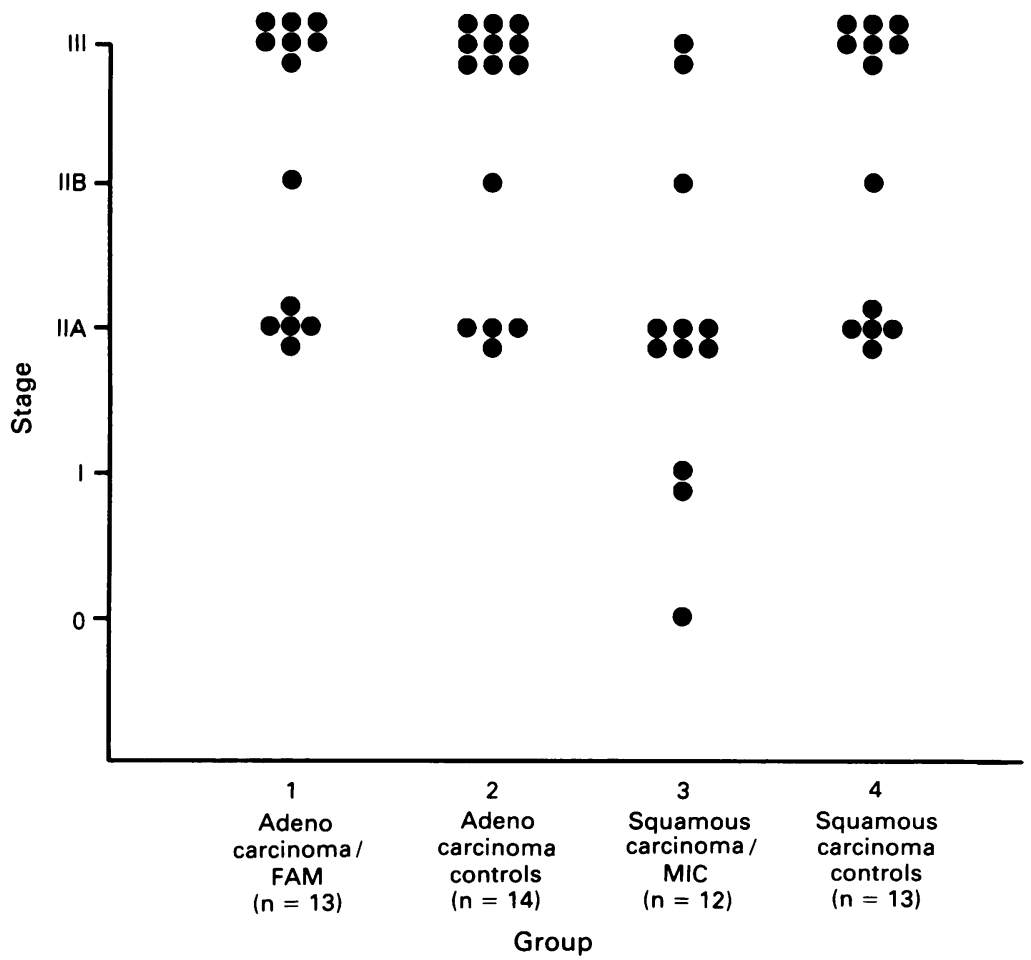

Figure 3 Staging of the resected specimens (by tumour invasion depth and local nodes) in the four patient groups.
MIC) with resected specimens in group 4 (untreated squamous carcinomas) showed that all 12 tumours in group 3 showed considerable differences from the untreated tumours. In one patient there was complete ablation of the tumour (fig 1B); in five there was pronounced tumour regression, with only minute foci of malignant cells scattered in the submucosa or muscularis propria, frequently surrounded by a mixed chronic inflammatory cell infiltrate (fig 2B). All 12 specimens showed a localised fibrotic response and a localised chronic inflammatory cell infiltrate, and 10 showed regeneration of the surface epithelium by a thin stratified squamous layer.

The staging of resected specimens by tumour and node status is shown in fig 3 . Groups 1 and 2 showed a similar range of staging, but group 3 patients showed a lower staging when compared with group 4 controls.

By quantitative analysis the mean proportion of tumour to stroma in the adenocarcinomas treated with FAM (group 1) was $73 \cdot 25 \%$ (CI 10.94). This did not differ significantly from that of the untreated adenocarcinomas (group 2) which was $71.56 \%$ (CI 12.58$)$. The mean value for the proportion of tumour to stroma in the group of squamous carcinomas treated with MIC (group 3) was $32.54 \%$ (CI 16.46) compared with $64.44 \%$ (CI 9.13) in the untreated cases. This difference was significant $(p<0.01)$ (fig 4). In this group there appeared to be two clearly distinguishable subgroups; one group of six patients (group 3a) where more than $50 \%$ of the field consisted of tumour and another group of six patients (group 3b) where less than 19\% of the field was occupied. The differentiation of the pretreatment biopsy specimens in these two subgroups was as follows: group 3a contained two poorly, one moderately, and three well differentiated tumours; group $3 \mathrm{~b}$ contained two poorly, one moderately, and three well differentiated tumours. Numbers are too small for formal statistics but group $3 \mathrm{~b}$ showed a tendency to better survival at one year-five out of six surviving, with the sixth a postoperative death, compared with three survivors out of six in group 3a, one death being unrelated and two due to recurrence of tumour.

\section{Discussion}

The study has shown that qualitative and quantitative responses to chemotherapy can be observed histopathologically in oesophageal cancer. Qualitative and morphometric techniques confirmed that the treatment of adenocarcinomas with FAM (group 1) was much less effective than treatment of squamous carcinomas with MIC (group 3).

Qualitative differences were seen following both chemotherapeutic regimens but were minor in group 1 compared with those in group 3, in both numbers of responders and extent. Previous light microscopic studies have described chemotherapeutic response in terms of the presence or absence of microscopic tumour, necrotic tumour, ${ }^{2}$ or of necrosis only. ${ }^{3}$ 


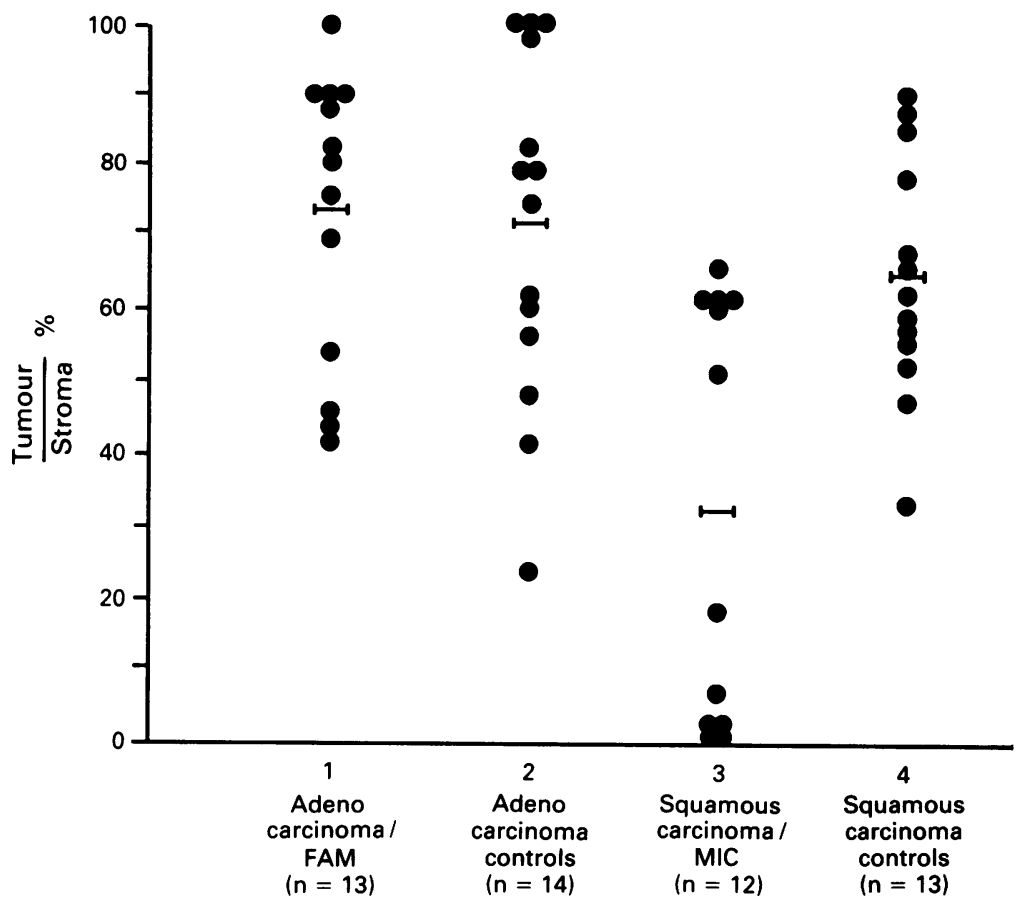

Group

Figure 4 Scatter diagram showing the proportion of tumour area to area of background stroma as a percentage for individuals in the four patient groups. Mean values are shown. Group 1 versus group 2, not significant. Group 3 versus group 4, significant $(p<0.01)$.

There has been one electron microscopic study reporting chemotherapeutic effect in terms of necrotic features of nuclei. ${ }^{4}$ Xian et al observed tumour necrosis and a stromal lymphoid response in oesophageal squamous carcinoma treated with traditional Chinese herbs. Like them, we also noted the frequent presence of a chronic mixed inflammatory cell infiltrate surrounding tumour areas after chemotherapy, but necrotic tumour areas were seen in only two of 13 cases in group 1 and one of 12 cases in group 3. Persisting tumour cells appeared viable even with tumour regression to small aggregates of cells (in group 3). It may be that during the chemotherapy treatment period of six weeks there was tumour cell death followed by in inflammatory response. Islands of inflammatory exudate were seen as haloes surrounding tumour islands in resected specimens from the treated groups. Healing of areas previously occupied by tumour appeared to be by fibrosis with overlying surface re-epithelialisation.

Routine histological examination of a resected specimen can determine complete response to chemotherapy, although sampling errors are possible unless serial sections are taken through the entire specimen. Assessment of partial response in a resected specimen by qualitative examination alone is more difficult. The Japanese system of reporting the histopathological response to chemotherapy in oesophageal tumours ${ }^{10}$ relies on the estimation of the proportion of necrotic to viable tumour cells. Their response grade Ef1 has one third of the tumour occupied by degenerate tumour cells; grade Ef2 is a degenerating tumour occupied by one third viable cells and grade Ef3 is a completely necrotic tumour with no viable cells. We consider this methodology to be inappropriate as in our experience reduction of tumour bulk in a healing oesophagus leaves small foci of apparently viable tumour with no necrotic areas. The presence of degenerate and necrotic tumour seen in other studies, and occasionally in this study, could be secondary to local ischaemia rather than representing a direct effect of chemotherapy. This discrepancy in results is possibly due to tumours being studied at different intervals following chemotherapy or because more of their data relate to tumours treated by radiotherapy. It is not possible to determine this as the necessary details are not stated in the publication. ${ }^{10}$

The morphometric technique used in this study quantitates a partial response to chemotherapy by measuring the actual ratio of tumour to background stroma in the treated groups when compared with the untreated groups. Quantitatively, the proportion of tumour to stroma was not significantly reduced by FAM chemotherapy in adenocarcinoma, despite minor qualitative changes seen in nine out of the 13 patients. The MIC regimen, however, significantly reduced the proportion of tumour to stroma in squamous tumours. In these patients there appeared to be two clearly divided subgroups in terms of degree of response. The numbers are small but it could be that these two subgroups represent a different susceptibility to MIC. The degree of differentiation of the tumour found at pretreatment biopsy bore no relation to susceptibility. Good quantitative responders, however, showed a tendency to improved survival at one year.

The similar staging in the treated and untreated adenocarcinomas confirms the status of group 2 cases as representative controls for group 1, bearing in mind the low level of histopathological response. When comparing the treated squamous carcinomas with their controls, the former showed a lower staging. This is of interest since staging of IIA and better (nine out of 12) represents local node negative patients with invasion into the adventitia or less. This could indicate a reduction in staging as a result of the chemotherapy but cannot be proved in the individual patient as resection is only performed once. The assumption of a chemotherapeutic effect of MIC on squamous carcinoma staging is reasonable as the observed reduction in the ratio of tumour to stroma provides indirect confirmatory evidence. The fact that there was no such lowered staging or reduction in tumour to stroma ratio in the adenocarcinomas treated with FAM also tends to corroborate this.

In conclusion, histopathological changes attributable to chemotherapy can be observed in oesophageal carcinoma. The response of squamous carcinoma to MIC chemotherapy is histologically more evident than that of adenocarcinoma to FAM chemotherapy. Qualitative observation of these changes may provide useful information about the response to chemotherapy, but it is of necessity imprecise and subject to observer bias. Quantitative analysis, particularly in relation to the propor- 
tion of tumour to stroma, is a simple technique which is more accurate and extends our ability to evaluate objectively the effect of chemotherapy on squamous carcinoma of the oesophagus.

This study was supported by the Oesophageal Cancer Research Appeal (OCRA), Birmingham, England.

1 Kelsen D. Neodjuvant therapy of esophageal cancer. Can $\mathcal{F}$ Surg 1989;32:410-14.

2 Hilgenberg AD, Carey RW, Wilkins EW, Choi NC, Mathisen DJ, Grillo HC. Preoperative chemotherapy, surgical resection, and selective postoperative resection, and selective postoperative therapy for squaThorac Surg

3 Hanaoka T, Nabeya K, Onozawa K, KobayashiY. Studies on preoperative treatment of esophageal cancer and prognosis of markedly effective cases. In: Siewart $\mathrm{AH}$, eds. Diseases of the oesophagus. Berlin: Springer-
Verlag, 1988:323-5

4 Iwatsuka M, Yoshida M. A study of the clinicopathological effects of chemotherapy for human esophageal carcinoma. In: Siewart JR, Holscher $\mathrm{AH}$, eds. Diseases of the oesophagus. Berlin: Springer-Verlag, 1988:319-22.

5 Xian M, Hayashi K, Lu J, Awai M. Efficacy of traditional chinese herbs on squamous cell carcinoma of the esophachinese herbs on squamous cell carcinoma of the esophagus: histopathologic analys

6 Walker SJ, Allen SM, Steel A, Cullen MH, Matthews HR. Assessment of the response to chemotherapy in oesophageal cancer. Eur f Cardiothorac Surg 1991;5:519-22.

7 Walker SJ, Allen SM, Steel A, Cullen MH, Matthews HR. A phase II study of 5-fluorouracil, adriamycin, and mitomycin in adenocarcinoma of the oesophagus. Clin Oncol 1991;3:318-22.

8 Matthews H, Walker S, Steel A, Cullen MH. Mitomycin, ifosfamide and cisplatin: an effective preoperative treatment for oesophageal carcinoma. Br 7 Cancer 1990;62: 497-8.

9 Hermanek P, Sobin LH. UICC TNM classification of malignant tumours. Berlin: Springer-Verlag, 1987.

10 Japanese Society for esophageal Diseases. Guidelines for the clinical and pathologic studies on carcinoma of the esophagus. Fpn $\mathcal{f}$ Surg 1976;6:69-86. 